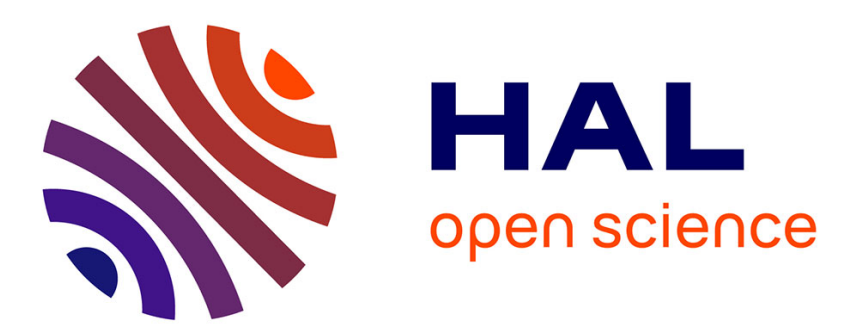

\title{
Enjeux de l'intégration des espaces naturels littoraux dans la gestion des risques liés à la mer
}

\author{
Camille Salmon, Virginie Duvat
}

\section{To cite this version:}

Camille Salmon, Virginie Duvat. Enjeux de l'intégration des espaces naturels littoraux dans la gestion des risques liés à la mer. Risques et résilience des territoires: apports de la notion de résilience à la gestion des risques, SHF, Oct 2017, Marne la Vallée, France. hal-01634357

\section{HAL Id: hal-01634357 https://hal.science/hal-01634357}

Submitted on 14 Nov 2017

HAL is a multi-disciplinary open access archive for the deposit and dissemination of scientific research documents, whether they are published or not. The documents may come from teaching and research institutions in France or abroad, or from public or private research centers.
L'archive ouverte pluridisciplinaire HAL, est destinée au dépôt et à la diffusion de documents scientifiques de niveau recherche, publiés ou non, émanant des établissements d'enseignement et de recherche français ou étrangers, des laboratoires publics ou privés. 


\title{
Enjeux de l'intégration des espaces naturels littoraux dans la gestion des risques liés à la mer
}

\author{
Camille SALMON ${ }^{1}$, Virginie DUVAT ${ }^{1}$
}

${ }^{1}$ UMR LIENSs 7266, Université de la Rochelle-CNRS - 2 rue Olympe de Gouges, 17000 La Rochelle, France -
camille.salmon@univ-lr.fr

En se basant sur deux études de cas situées dans l'outre-mer français, cet article traite de l'intégration des espaces naturels littoraux dans la gestion des risques liés à la mer. L'étude des impacts de et de la résilience aux cyclones Oli (février 2010) à Tubuai (Polynésie française) et Bejisa (janvier 2014) à la Réunion, a mis en évidence le rôle majeur de la dégradation de la zone tampon (systèmes plages-dunes dans notre étude) dans l'augmentation de la vulnérabilité. Cinq processus de dégradation sont analysés : la contraction (diminution de la surface), la fragmentation des cellules hydro-sédimentaires par des aménagements côtiers, la fixation du trait de côte par des ouvrages de défense, le défrichement et la modification de la végétation et enfin l'extraction de matériaux coralliens sur les plages. Ces phénomènes ont pour conséquence la perturbation de la fonction d'amortissement des vagues et du vent, ce qui au final augmente l'endommagement des enjeux humains lors de la survenue d'un événement météo-marin extrême. Ces résultats ont des implications concrètes pour favoriser la résilience des territoires face aux risques liés à la mer : (1) mener une politique de protection et/ou de restauration des espaces naturels littoraux, (2) considérer la cellule hydrosédimentaire comme l'échelle d'intervention pertinente, (3) mettre en œuvre des mesures différenciées qui prennent en compte le long terme, afin d'engager dès maintenant une politique d'adaptation au changement climatique.

Mots-clefs : zone tampon; résilience ; cyclones ; systèmes morpho-sédimentaires ; outre-mer.

\section{Taking into account the natural coastal systems}

\section{in coastal risk management}

\begin{abstract}
Based on two case studies located in French overseas territories, this presentation aims to investigate how to base coastal risk management policies on natural coastal system protection. The in-depth analysis of the impacts of and resilience to cyclones Oli in Tubuai Island (French Polynesia, Feb. 2010) and Bejisa in Reunion Island (Jan. 2014), raised the major role of natural buffers degradation (i.e. sand-dune systems) in increasing vulnerability. Five processes are analysed here: contraction (reduction in land area), fragmentation of sedimentary cells by coastal structures, shoreline fixation due to coastal defences, removal or modification of vegetation and finally, coral beach extraction. These phenomena alter the attenuation function of waves and wind, and finally increase damages when a coastal hazard occurs. This results have concrete implications in terms of enhancing resilience of territories to sea-related risks, enabling to: (1) design and implement conservation and restoration policies so as to strengthen the buffering capacity of sand-dune systems; (2) consider the sediment cell as the relevant scale for coastal risk management; (3) implement context-specific solutions that take into account long-term changes, so as to initiate a climate change adaptation policy.
\end{abstract}

Key words: buffer zone, resilience; tropical cyclones; morpho-sedimentary systems; French overseas territories.

\section{INTRODUCTION}

En complément des études sur la vulnérabilité, la notion de résilience a émergé et est devenue un élément porteur pour étudier les risques naturels, en particulier les aléas météo-marins. Elle permet de développer une réflexion holistique et systémique, c'est-à-dire prenant en compte toutes les composantes d'un système et leurs interactions, contrairement à une approche sectorielle [Berkes, 2007]. Elle invite également à adopter une approche dynamique (son évaluation se faisant généralement en mesurant la durée qu'un système met pour retrouver une situation "normale ») et prospective : en interrogeant la réponse à un aléa, elle invite à se projeter et à explorer des options pour faire face aux incertitudes futures [Berkes, 2007]. Ainsi, développer des études de cas d'une perturbation sur un territoire donné, sous forme de retour d'expérience par exemple, est pertinent pour apporter des connaissances empiriques sur: (1) les différentes modalités de la résilience d'un système ; (2) les facteurs jouant un rôle important dans l'expression ou non de cette résilience, ainsi que leurs temporalités. 
En parallèle, de nombreux travaux s'intéressent au rôle de zone tampon des espaces naturels littoraux et marins : récifs coralliens, herbiers, mangroves, systèmes plages-dunes, et marais maritimes [Cooper et McKenna, 2008 ; Debaine et Robin, 2012 ; Arkema et al., 2013 ; Spalding et al., 2014 ; Rao et al., 2015 ; Eliff et Silva, in press]. Alors que les solutions d'ingénierie lourde sont dans de nombreux cas indispensables pour protéger les côtes, elles ne sont pas toujours souhaitables, car elles induisent des coûts élevés et dégradent l'environnement [Arkema et al., 2013 ; Spalding et al., 2014], d'où la volonté d'étudier des solutions alternatives. La préservation d'espaces naturels en bon état de santé apparait donc comme un élément clé pour favoriser la résilience des territoires face aux aléas météo-marins tels que les cyclones, grâce à leur rôle d'amortissement des vagues et du vent. Néanmoins, ces travaux font le constat de leur dégradation, sous l'effet de facteurs locaux (urbanisation, pollution) et globaux (acidification des océans, élévation du niveau marin), ce qui entraîne une perturbation de leur fonction de protection.

L'objectif de cet article est d'analyser les impacts de la dégradation de la zone tampon sur l'exposition et la vulnérabilité des enjeux humains aux aléas météo-marins, à travers l'étude de la situation de deux îles tropicales de l'outre-mer français, Tubuai et la Réunion. Cet article se concentre sur la zone tampon terrestre, constituée d'un système plage-dune, qui s'étend du pied de plage aux premiers enjeux en dur (routes et bâti). Elle comprend une partie active, la plage, et une partie stabilisée, l'arrière-plage ou la dune, séparées selon les cas par la limite de la végétation (dite « ligne de végétation ») ou par des ouvrages de défense [Duvat et al., 2016b]. Cet article présentera d'abord le contexte et les caractéristiques de la zone tampon étudiée, puis son évolution récente et les processus qui régissent sa dégradation. Dans un troisième temps, nous analyserons les conséquences de la dégradation de la zone tampon lors de la survenue de cyclones, à travers les exemples de Bejisa à la Réunion (janvier 2014) et de Oli à Tubuai (février 2010). Enfin, nous discuterons des implications des résultats obtenus pour la gestion des risques et l'adaptation au changement climatique.

\section{CONTEXTE}

Les îles de la Réunion et de Tubuai sont deux îles volcaniques situées globalement à la même latitude $\left(21^{\circ} \mathrm{S}\right.$ et $\left.23^{\circ} \mathrm{S}\right)$, mais dans deux bassins océaniques différents (Indien et Pacifique) (figure 1A-B). Ces deux îles constituent des cas d'étude pertinents sur la thématique des risques, et représentatifs d'un certain nombre de territoires d'outre-mer français. Leurs modalités de peuplement et leurs densités de population sont très différentes : alors que la Réunion (2512 km²) atteint environ 835000 hab. (soit $332 \mathrm{hab} . / \mathrm{km}^{2}$ ) et connaît une très forte urbanisation littorale depuis les années 1970, Tubuai $\left(45 \mathrm{~km}^{2}\right)$ ne compte que $2322 \mathrm{hab}$. en 2012 (soit $52 \mathrm{hab} . / \mathrm{km}^{2}$ ) et connait une faible croissance démographique, Ces deux cas permettent ainsi d'interroger l'évolution de la zone tampon dans des configurations humaines contrastées.

Cette étude se concentre sur les zones les plus affectées par les cyclones étudiés, soit la commune de SaintPaul à l'ouest de la Réunion (figure 1C) et la partie nord de Tubuai (figure 1D) - communes de Mataura et Taahuaia. A la Réunion, la zone tampon est divisée en une succession de petites cellules hydrosédimentaires, séparées par des portions de côte rocheuse. Elle est composée d'un littoral alluvionnaire au nord (figure 2A) - constitué de plages et de cordons de sables et galets volcaniques bordant un cône-delta - et d'un littoral corallien au sud, du cap la Houssaye à la passe de Trois Bassins, composé de plages, cordons et dunes de sables et débris coralliens adossés à de petites plaines littorales. Le système récifal est constitué au nord de plateformes et bancs récifaux (Cap La Houssaye-Grands Fonds) et il s'élargit progressivement vers le sud pour atteindre le stade de récif frangeant (Ermitage-La Saline) (figure 3A), avec une largeur de $500 \mathrm{~m}$ [Cazes-Duvat et Paskoff, 2004]. A Tubuai, la zone tampon constitue une cellule sédimentaire unique, en raison de l'absence de promontoires rocheux interrompant le transit côtier. Elle est constituée de plages et cordons de sables coralliens, qui isolent à l'ouest une vaste zone marécageuse, et sont séparés par de petites embouchures, nombreuses dans le sud de l'île. Elle s'adosse à une étroite plaine littorale (figure 2B) enserrant un relief montagneux culminant à $419 \mathrm{~m}$ (Mont Taita'a). Le récif a atteint le stade de récif barrière, avec un lagon dont la largeur dépasse par endroits $4 \mathrm{~km}$. Dans les deux îles, c'est principalement dans la plaine côtière que la population et les activités humaines se sont implantées.

Tubuai et La Réunion sont particulièrement exposées à l'aléa cyclonique, l'occurrence moyenne d'un événement étant estimée respectivement à 1 et 2,8 cyclones par décennie [Duvat et al., 2016a ; Etienne, 2012]. Le cyclone Bejisa (catégorie 3) est passé sur la Réunion le 2 février 2010 (figure 1A), avec des rafales à $140 \mathrm{~km} / \mathrm{h}$ sur le littoral ouest et sud. Le pic d'intensité de la houle s'est produit vers midi, avec des hauteurs significatives estimées à $7,5 \mathrm{~m}$ et une période inférieure à 12 secondes [Aunay et al., 2014]. Le cyclone Oli (catégorie 4) est passé sur Tubuai dans la nuit du 4 au 5 février 2010 (figure 1B), avec des rafales de vent à 
$169 \mathrm{~km} / \mathrm{h}$, des vagues au large d'une hauteur significative estimée à $15-16 \mathrm{~m}$ et une période entre 13 et 15 secondes. La surcote atmosphérique a été estimée à $0,80 \mathrm{~m}$ dans le lagon [Lecacheux et al, 2013].

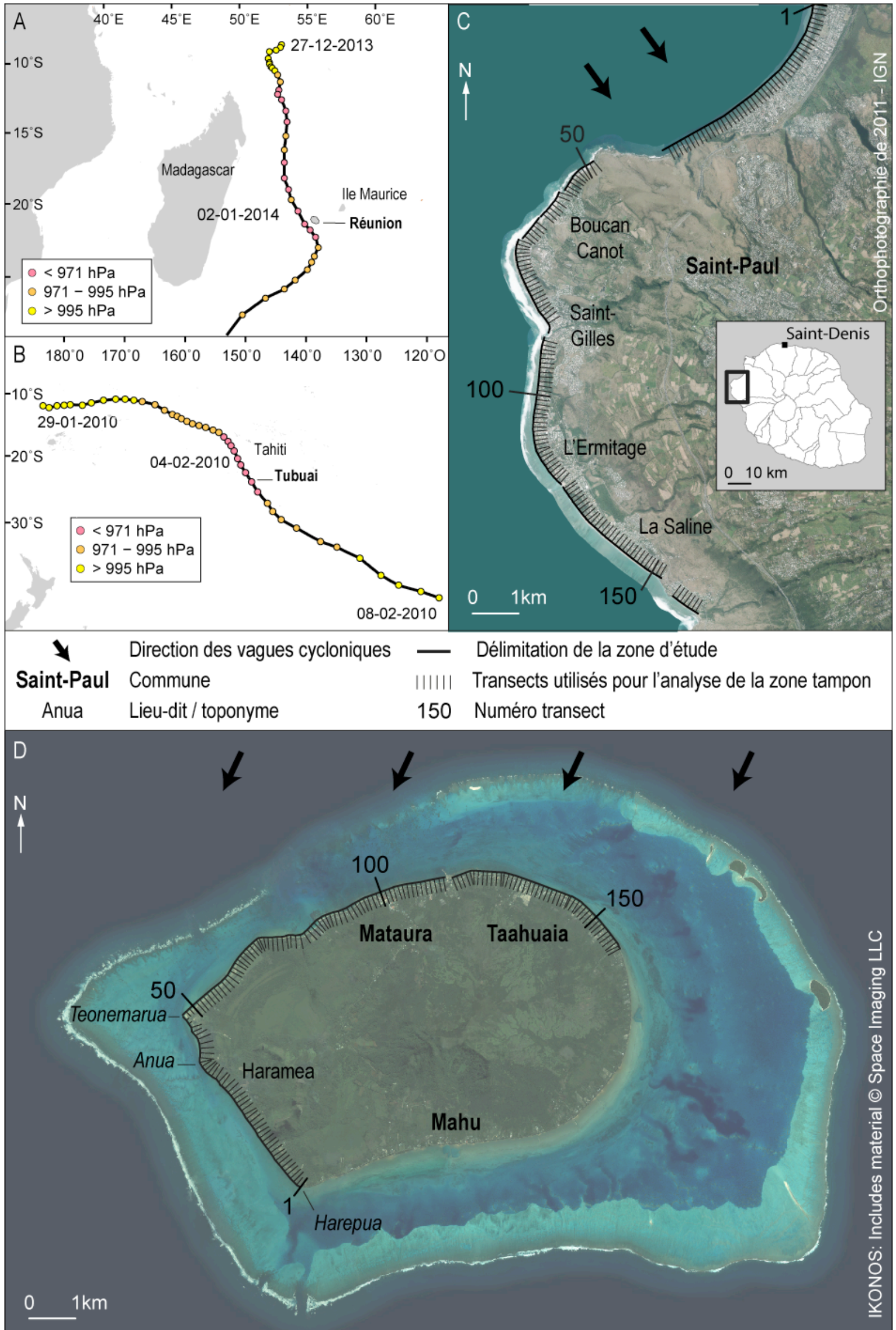


Figure 1 - A) Localisation de la Réunion dans l'ouest de l'océan Indien et trajectoire du cyclone Bejisa ; B) Localisation de Tubuai dans l'océan Pacifique et trajectoire du cyclone Oli. C) Présentation de la zone d'étude à la Réunion : le littoral de la commune de Saint-Paul, de la rivière Bernica à la passe de Trois-Bassins. D) Présentation de la zone d'étude à Tubuai : de la pointe de Harepua au village de Taahuia. Des transects générés tous les $100 \mathrm{~m}$ le long du trait de côte ont permis d'analyser la surface de la zone tampon à différentes dates. Source pour les trajectoires des cyclones : http://www.bom.gov.au/cyclone/history/tracks/

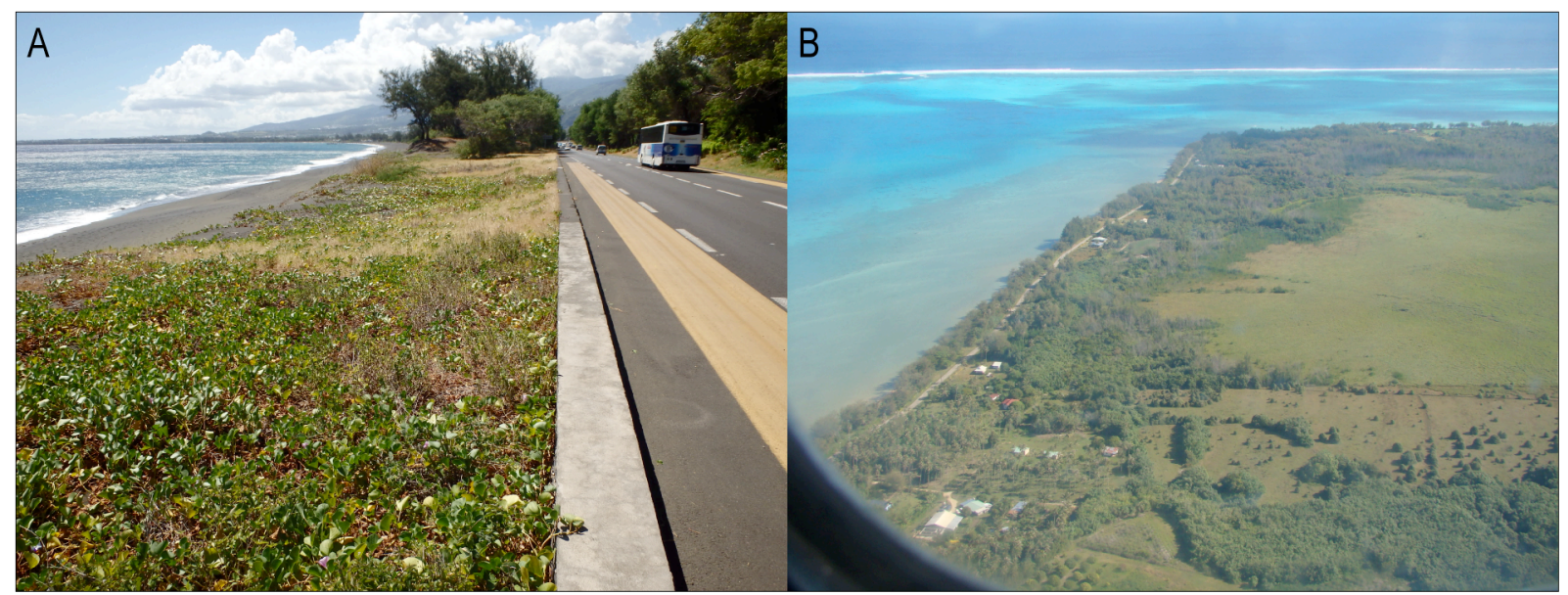

Figure 2 - Photographies de la zone tampon à la Réunion et à Tubuai. A) Sud de la baie de Saint-Paul. On voit, au premier plan, la zone tampon stabilisée correspondant à l'arrière-plage végétalisée, interrompue par la route côtière (= limite des enjeux), et au second plan, la zone tampon active, correspondant à une plage de sable volcanique. B) Photographie aérienne de Tubuai, montrant la plaine littorale sur laquelle la route a été construite au premier plan, et le lagon et récif-barrière en second plan.

\section{MATÉRIEL ET MÉTHODES}

Cet article s'appuie principalement sur des résultats issus des programmes de recherche Réomers et Storisk [Duvat et al., 2016a; Duvat et Magnan, 2016], et sur l'étude réalisée par Etienne (2012) sur Tubuai. L'évolution de la surface de la zone tampon a été analysée, à la Réunion, entre 1950 et 2011 (période de 61 ans) et, à Tubuai, entre 1982 et 2006 (période de 24 ans), à partir de photographies aériennes et d'images satellites. Des transects générés sous DSAS tous les $100 \mathrm{~m}$ ont permis de découper la zone tampon en «boîtes» de dimension comparable (cf. figure 1C-D), afin d'affiner l'échelle d'analyse (ce découpage a notamment permis de sortir les différents graphiques des figures 3 et 4). Les autres données relatives à la zone tampon (sur sa fixation, sur la végétation, etc.) ont été créées à partir de l'analyse des images et d'observations terrain. La cartographie des impacts sur le bâti et sur les infrastructures a été réalisée à partir d'entretiens auprès des habitants et des acteurs publics. Le recouvrement du pied de plage par la végétation a limité l'extension vers l'est de la zone d'étude à Tubuai.

\section{RESULTATS}

\section{Etude de $\operatorname{cas}^{\circ}{ }^{\circ} 1$ : le littoral ouest de la Réunion}

\section{IV.1.1 Evolution récente de la zone tampon}

Le littoral de Saint-Paul a connu entre 1950 et 2011 un phénomène de contraction de la zone tampon, avec une perte totale de 51,5 ha (39\%). La surface totale de la plage (ou zone tampon active) est passée de 61,3 ha à 42,8 ha $(-30 \%)$, et celle de l'arrière-plage végétalisée (ou zone tampon stabilisée) est passée de 72,2 ha à 39,2 ha $(-46 \%)$. En 2011, la surface moyenne de la zone active (par " boîte ») était d'environ 0,27 ha (contre 0,38 ha en 1950) et celle de la zone stabilisée était de 0,25 ha (contre 0,46 ha en 1950). Si cette contraction est plutôt homogène pour la zone tampon active (ce que nous montre le graphique $\mathrm{B}$ de la figure 3), elle est plus contrastée pour la zone tampon stabilisée. Le graphique $C$ permet de distinguer trois secteurs relativement épargnés par l'urbanisation : le nord de la baie de Saint-Paul (transects 1-17), le secteur du Cap Homard (transects 61-64) et la dune de l'Ermitage (transects 100-120). A l'inverse, dans les secteurs restants, ce même graphique montre que les pertes de surface ont été très importantes, comme l'illustre le cas 
de la Saline (transects 127-147). De plus, le fonctionnement en cellule hydro-sédimentaire a parfois été perturbé par l'implantation d'aménagements transversaux. C'est le cas du port de plaisance de Saint-Gilles, construit au début des années 1970 et agrandi dans les années 1990, qui a interrompu le transit côtier nordsud et divisé en deux la cellule hydro-sédimentaire originelle [Duvat et al., 2016b]. Cela a entraîné l'engraissement de la plage des Brisants en amont-dérive du port et l'érosion de la plage des Roches Noires en aval-dérive [Cazes-Duvat et Paskoff, 2004]. Le graphique suivant (figure 3D) montre que le trait de côte a subi une artificialisation importante entre 1950 et 2011. En effet, la longueur de linéaire côtier équipé d'ouvrages de défense est passée de $0,98 \mathrm{~km}$ à $7,09 \mathrm{~km}$, même si la plupart de ces ouvrages sont artisanaux et ont été édifiés par les riverains [Duvat et al., 2016b]. Ils posent deux problèmes principaux dans la capacité de réponse du système : 1) ils font obstacle aux échanges transversaux de sédiments, entre la plage et l'arrière-plage ou la dune ; 2) ils accentuent l'érosion des plages en augmentant la réflexion des vagues. Cette perturbation du littoral s'accompagne par une dégradation de la végétation, du fait de défrichements ayant engendré une contraction de la couverture végétale, et d'une modification de la nature de la végétation. Le graphique E montre que dans les zones urbanisées, les espèces indigènes ont quasiment disparu, alors qu'elles résistent davantage aux vents violents et aux vagues que les espèces introduites [Duvat et al., 2016a]. Enfin, le nettoyage mécanique des plages coralliennes (Ermitage), qui a eu cours de 1979 à 1988 et a consisté à en retirer les débris coralliens, a grevé leur budget (abaissement de 1,5 cm/an) et ainsi contribué à leur érosion [Troadec, 2003].

\section{IV.1.2 Conséquences de la dégradation de la zone tampon sur l'augmentation de la vulnérabilité aux aléas météo-marins}

La modification des caractéristiques de la zone tampon engendre la diminution, voire l'annihilation par endroits de sa capacité d'amortissement. La contraction de cette zone tampon s'accompagnant généralement de la croissance des enjeux humains, de par l'implantation de nouveaux enjeux plus près du trait de côte et dans des zones basses [Duvat et al., 2016b], on assiste au final à une augmentation de la vulnérabilité des enjeux face aux aléas météo-marins extrêmes. Le passage du cyclone Bejisa a fait ressortir cette situation préoccupante. Les levés réalisés entre le 11 et 15 janvier 2014 sur $9,1 \mathrm{~km}$ de linéaire côtier indiquent que 15 ouvrages de défense (dont quelques ouvrages publics) ont été endommagés (la figure $3 \mathrm{~F}$ montre que les dégâts se situent principalement dans les cellules 2 à 5) et que 33 bâtiments et infrastructures ont subi des impacts modérés (22) à forts (11). La figure $3 \mathrm{G}$ montre que les principales zones d'impacts ont été le centre de la baie de Saint-Paul, le Cap la Houssaye où la route côtière a été sévèrement endommagée, et surtout la zone du port de Saint-Gilles, où l'inondation par la ravine de Saint-Gilles et la submersion (avec des vagues peu réfractées en raison de la présence de la passe) se sont combinées. Les impacts du cyclone Bejisa ont donc été importants d'une part, sur les sections de côte exposées (orientées NO), là où le récif est absent, étroit ou interrompu par une passe, et d'autre part, là où la zone tampon s'est contractée depuis 2011, sous l'effet du recul de la plage (implantation d'ouvrages de défense) ou de l'empiètement de l'urbanisation sur l'arrière-plage ou la dune végétalisée.

\section{Cas $\mathbf{n}^{\circ} 2$ : le cyclone Oli à Tubuai}

\section{IV.2.1 Evolution récente de la zone tampon}

La période d'analyse est ici plus réduite que dans le cas précédent (1982-2006). De fait, la surface de la zone tampon totale en 1982 était déjà limitée $(69,25$ ha), mais celle-ci a continué à baisser pour atteindre 56,04 ha en 2006 (- $19 \%)$. La surface totale de la plage est passée de 18,40 ha en 1982 à 10,61 ha en $2006(-43 \%)$ et sa surface moyenne (par «boîte ») de 0,12 ha à 0,09 ha. A noter la disparition de la zone tampon active sur une grande partie de la façade occidentale de l'île et sa contraction au-devant de la zone de l'aéroport (figure 4A). Dans les zones habitées de Mataura et Taahuai, les changements sont moins marqués car les surfaces étaient déjà réduites en 1982. L'arrière-plage végétalisée a connu une évolution plus modérée, sa surface totale passant de 50,84 ha en 1982 à 45,43 ha en $2006(-10 \%)$ et sa surface moyenne (par « boîte ») de 0,35 ha à 0,30 ha. Deux zones ont connu une réduction importante de leur surface : entre les pointes d'Anua et Teonemarua (transects 41-44) et à proximité de l'aéroport (transects 67-70). Partout ailleurs, les surfaces étaient là-aussi déjà très limitées en 1982 (figure 4B). L'île de Tubuai a donc connu une forte contraction de la zone tampon, mais qui s'est opérée avant 1982, avec la construction de cette route côtière. Ce constat de forte dégradation est à mettre en perspective avec la faible densité de population de l'île $\left(52 \mathrm{hab} / \mathrm{km}^{2}\right)$, ce qui montre que le facteur démographique ne contrôle pas toujours l'augmentation de l'exposition des populations dans les zones à risques. 


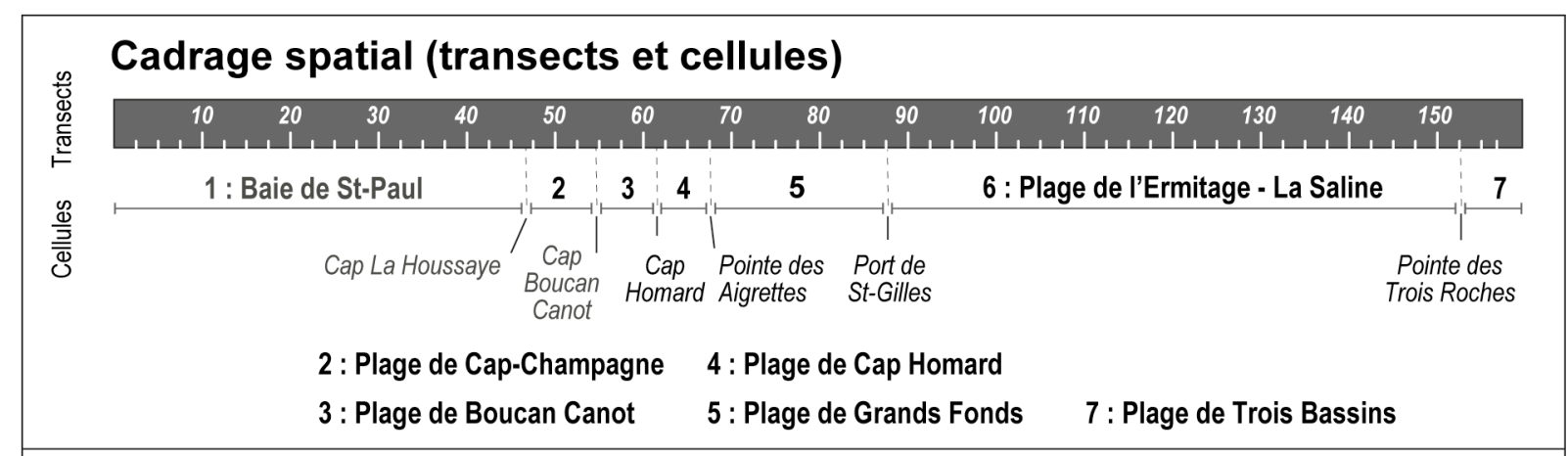

\section{Caractéristiques et évolution récente de la zone tampon}

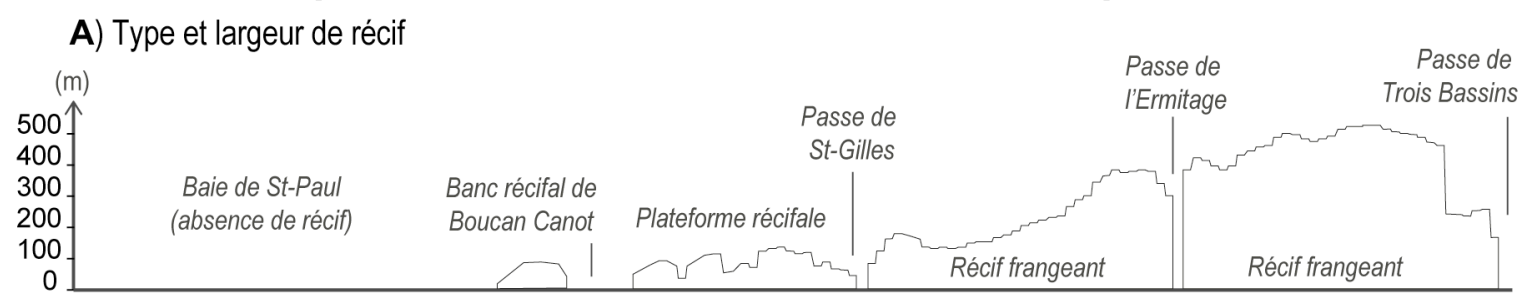

B) Evolution de la surface de la zone tampon active

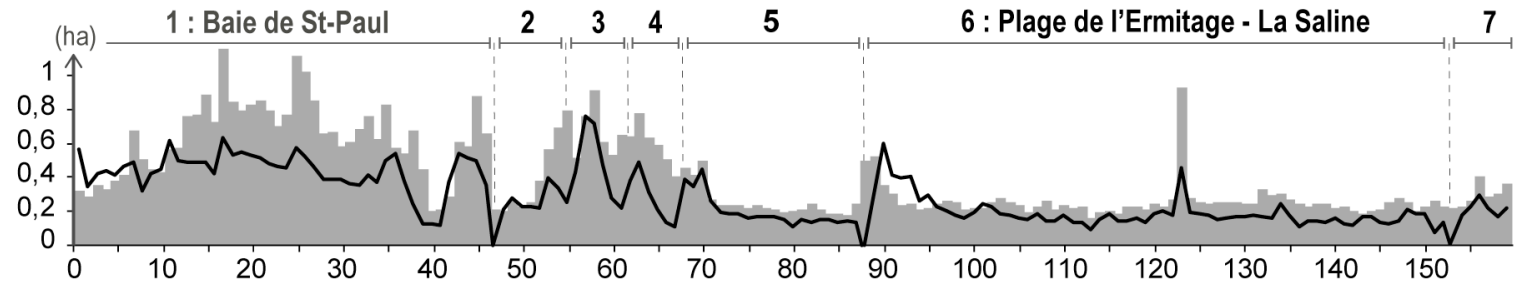

C) Evolution de la surface de la zone tampon stabilisée
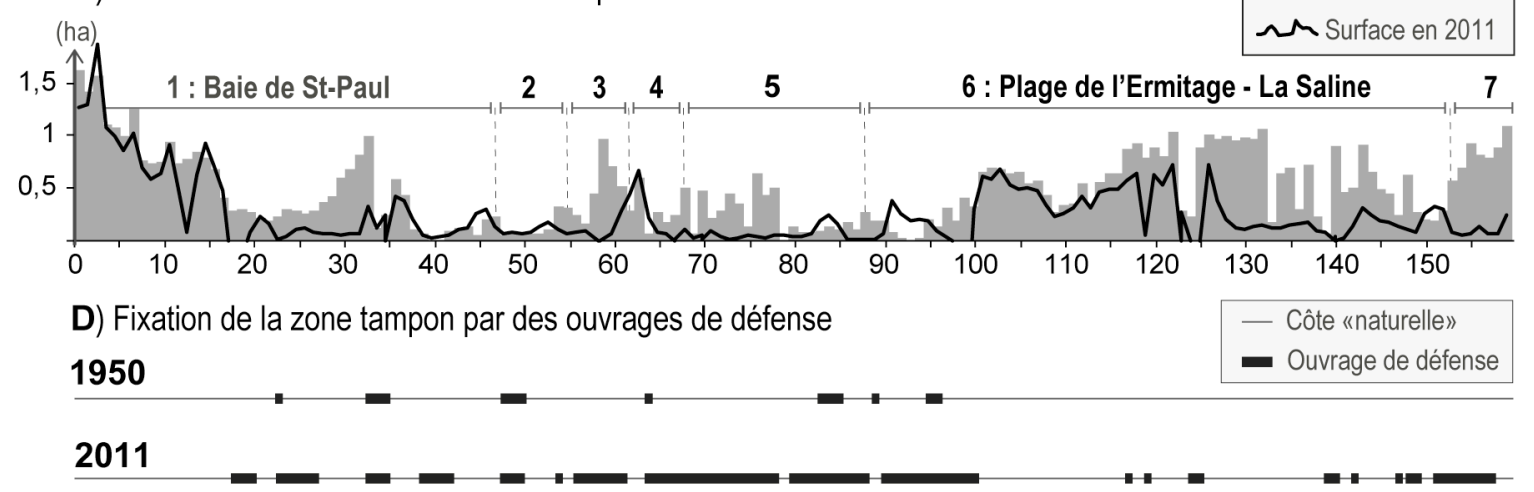

E) Caractéristiques de la végétation

$\begin{array}{cccc}\text { In/I } & \text { In/I } & \text { In/I } \\ \text { - Principales zones de végétation } & \text { I Espèces introduites dominantes } & \text { In/l Mélange d'espèces introduites et indigènes }\end{array}$

\section{Endommagement lié à Bejisa}

F) Impacts sur les ouvrages de défense

- Ouvrage de défense endommagé

G) Impacts sur le bâti et les infrastructures

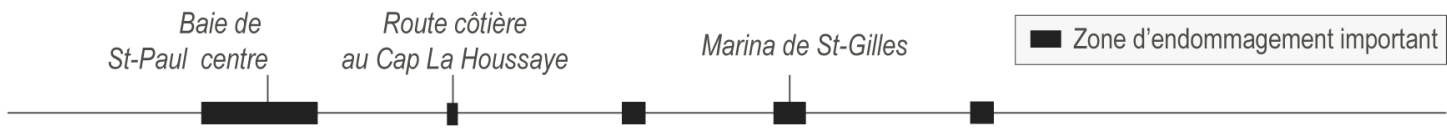

Figure 3 - Relation entre les caractéristiques de la zone tampon et l'endommagement lié au cyclone Bejisa. Le cadrage spatial rappelle le découpage de la zone d'étude en cellules hydro-sédimentaires, avec des transects générés tous les 100 
$\mathrm{m}$ (cf. figure 1 pour la localisation des transects). Il s'applique à l'ensemble de la figure. Le bloc suivant présente les caractéristiques et l'évolution récente de la zone tampon : A) Le contexte récifal est indiqué (type de récif + largeur), car il constitue la partie marine de la zone tampon. Les graphiques $\mathrm{B}$ et $\mathrm{C}$ montrent l'évolution de la zone tampon active et stabilisée entre 1950 et 2011. Les transects et cellules sont indiquées de nouveau pour mieux situer ces évolutions. D) Evolution de sa fixation par des ouvrages de défense entre 1950 et 2011 et E) Caractéristiques synthétiques des principales zones de végétation présentes dans la zone d'étude. Enfin, le dernier bloc résume les principaux impacts du cyclone sur F) les ouvrages de défense et; G) le bâti et infrastructures.

A cela s'ajoute la fragmentation de la zone tampon, générée par l'implantation d'aménagements qui bloquent les échanges sédimentaires. On observe ainsi des discontinuités à la fois dans la plage et l'arrière-plage (les graphiques $\mathrm{A}$ et $\mathrm{B}$ de la figure 4 montrent de fait où se situent ces discontinuités). Concernant la fixation de la zone tampon, même si nous ne possédons pas de données précises sur les ouvrages de défense en 1982, la comparaison des images aériennes montre qu'il se sont démultipliés entre cette date et 2006, ce qui a contribué à l'abaissement et au recul des plages. Nous observons également que la végétation arborée a diminué. Il ne reste par endroits qu'une mince frange végétale, telle la ripisylve d'une rivière, impropre à absorber l'énergie de vagues cycloniques. De plus, les espèces introduites (Casuarina equisetifolia) sont moins résistantes que les espèces indigènes et empêchent ces dernières de se développer.

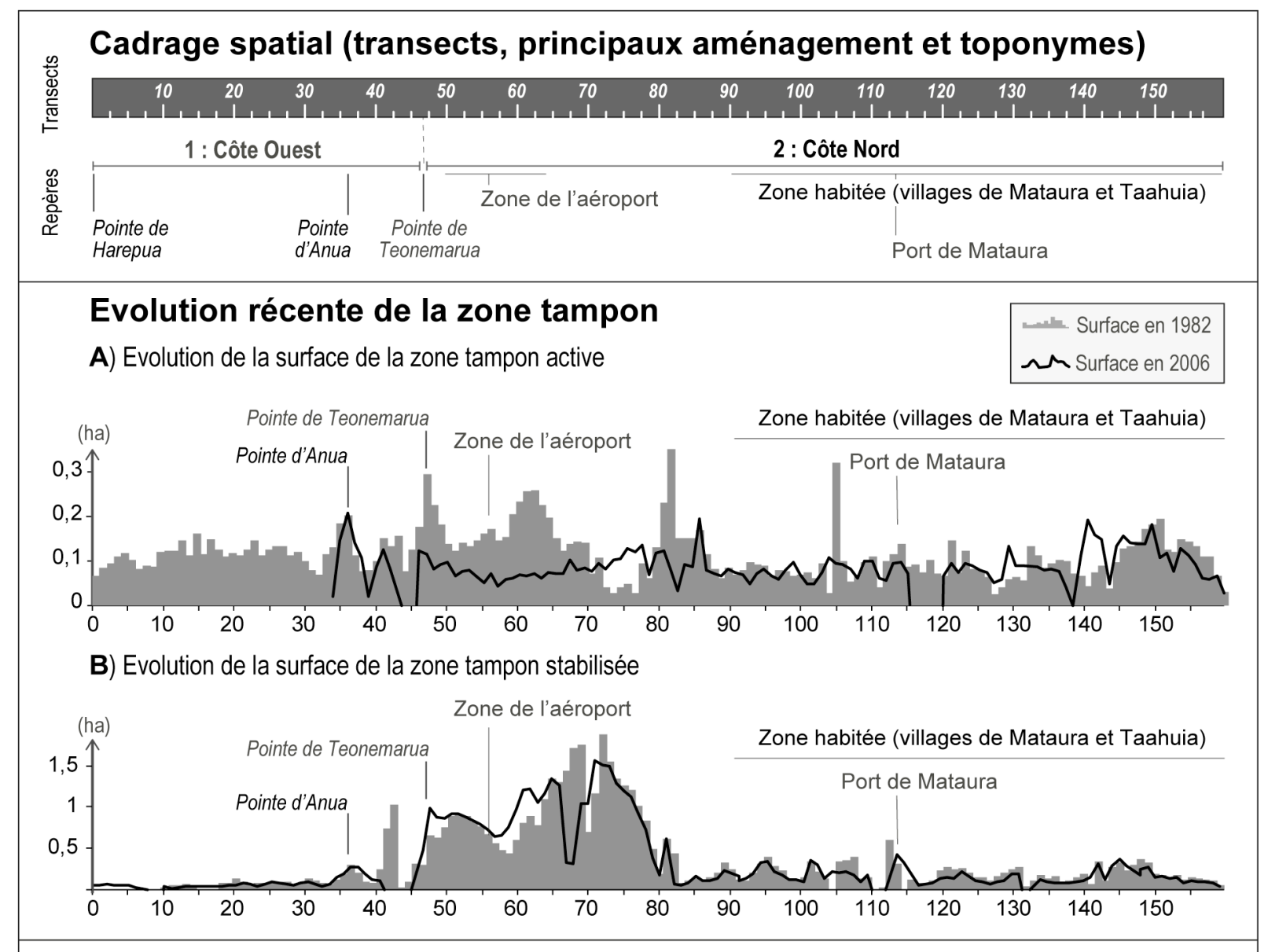

\section{Endommagement lié à Oli}

C) Impacts sur les routes

D) Impacts sur le bâti

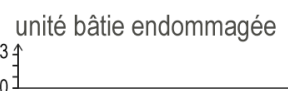

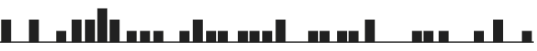

Figure 4 - Relation entre l'évolution récente de la zone tampon et l'endommagement lié au cyclone Oli. Le cadrage spatial rappelle le découpage de la zone d'étude en fonction des transects générés tous les $100 \mathrm{~m}$ (cf. figure 1 pour la 
localisation des transects). Il s'applique à l'ensemble de la figure. Le bloc suivant présente l'évolution récente de la zone tampon : Les graphiques A et B montrent l'évolution de la zone tampon active et stabilisée entre 1982 et 2006 . Les transects et cellules sont indiqués de nouveau pour mieux situer ces évolutions. Enfin, le dernier bloc résume les principaux impacts du cyclone sur C) les routes et ; D) le bâti.

\section{IV.2.2 Conséquences de la dégradation de la zone tampon sur l'augmentation de la vulnérabilité aux aléas météo-marins}

Dans le cas de Tubuai, la fonction d'amortissement de la zone tampon a également été perturbée par une urbanisation trop proche de la côte, ce qui accroît l'exposition et la vulnérabilité de la population, d'autant que la zone la plus densément peuplée est située sur la côte exposée aux houles cycloniques. Le cyclone Oli est considéré comme un cyclone exceptionnel de par son intensité. En témoignent les dégâts à la côte, avec une érosion généralisée des plages et une submersion marine sur toute la partie nord, jusqu'à $250 \mathrm{~m}$ dans les terres [Laurent et Varney, 2014]. La route côtière a été recouverte par des nappes d'épandage ou interrompue par la formation de crevasses (la figure 4C montre la localisation des portions de route concernées). Selon les estimations, $70 \%$ de l'habitat de l'île a été affecté par les vents violents et la houle [Etienne, 2012], principalement sur la côte nord. La figure 4D montre la distribution spatiale des impacts sur le bâti après Oli. Ceux-ci sont logiquement plus nombreux dans les zones habitées de Mataura et Taahuai, où encore une fois, la proximité de la route au trait de côte a entrainé l'installation de la population trop près de la mer. Contrairement à la Réunion, nous n'avons pas ici de données sur les ouvrages de défense endommagés. Ce que nous pouvons dire est que les blocs composant les cordons d'enrochement ont parfois été projetés par les vagues sur la route et les habitations, produisant des dégâts très importants [Etienne, 2012], principalement là où la zone tampon est très réduite.

\section{DISCUSSION}

Ces résultats donnent une clé de lecture intéressante des conséquences des changements qu'ont connu les littoraux au cours des dernières décennies sur l'exposition et la vulnérabilité des sociétés. En effet, ils mettent en évidence le lien entre les impacts des cyclones sur le bâti et les infrastructures, et les caractéristiques de la zone tampon (nature et évolution récente). La superposition des zones d'impacts avec l'évolution pluri-décennale de la zone tampon (cf. graphiques des figures 3 et 4) montre que les impacts sur les enjeux humains ont été les plus marqués là où la zone tampon a quasiment disparu, même si d'autres facteurs ont joué un rôle important, comme l'orientation de la côte par rapport à la direction des houles cycloniques, qui explique en grande partie la distribution spatiale et l'intensité des impacts des cyclones à la côte [Duvat et al., 2016a]. Le contexte récifal et d'une manière générale la bathymétrie, sont également des facteurs cruciaux : alors que le front récifal et le platier atténuent l'énergie des vagues [Elliff et Silva, in press], les passes favorisent leur propagation vers la côte et canalisent des courants érosifs pour les plages, comme on a pu l'observer à la Réunion. Il ressort donc de cette étude que le facteur « zone tampon » joue un rôle important dans la vulnérabilité des enjeux humains, ce qui peut aider à définir des stratégies pour le futur. Schématiquement, deux situations se dégagent :

1) la zone tampon encore fonctionnelle, dont les dimensions sont suffisamment importantes pour absorber les impacts négatifs d'une perturbation. La partie active est généralement résiliente : à la Réunion, une partie des plages de Saint-Paul a retrouvé un état d'équilibre un an après le passage de Bejisa, au nord de la baie de Saint-Paul et à Boucan Canot notamment (Duvat et al., 2016a). A Tubuai, Etienne (2012) a constaté une résilience importante des plages près de la pointe d'Anua quelques mois après le cyclone Oli, alors que les plages situées à Mataura ont continué à s'éroder.

2) la zone tampon très dégradée, à l'arrière de laquelle l'urbanisation s'est développée de manière forte (avec donc de nombreux enjeux humains à protéger près du trait de côte), et qui n'assure plus ou presque son rôle d'amortissement. On retrouve cette situation à Grands Fonds avec notamment à son extrémité méridionale le port de plaisance de Saint-Gilles, ou encore sur quelques portions de côte au nord-est de Tubuai, où la zone tampon - plage et arrière-plage cumulée -, est parfois inférieure à $20 \mathrm{~m}$ de largeur (transects 131-138).

A partir de ce constat, deux pistes au moins se dessinent, « travailler avec la nature en s'appuyant sur les zones tampons », et privilégier la protection lourde, lorsque l'option précédente n'est pas envisageable.

Lorsque la protection des espaces naturels parait difficile à envisager (cas de dégradation très avancée + forte urbanisation, que l'on retrouve en aval du port de Saint-Gilles à la Réunion, ou encore à Taahuai à Tubuai), il faudra privilégier la protection lourde, en mettant des moyens suffisamment importants pour 
construire des ouvrages adaptés et bien calibrés (et qui donc se dégraderont moins vite). Dans certains cas, la solution peut passer par une combinaison de solutions (douces + lourdes, par exemple un reprofilage de plage avec une revégétalisation de l'arrière-plage, associée à la mise en place de brise-lames pour réduire l'énergie des houles).

En revanche, la zone tampon encore fonctionnelle peut faire l'objet d'une politique de préservation (cf. le nord de la baie de Saint-Paul et l'Ermitage à la Réunion), et parfois de restauration. Cela passe dans un premier temps par : 1) la réduction des pratiques qui altèrent la résilience du système plage-dune. Dans ces deux exemples, une partie des matériaux apportés par le cyclone (sables et débris coralliens) a été extrait par l'homme, notamment pour la reconstruction, ne permettant pas à la topographie de s'exhausser. Les effets positifs que peut avoir un cyclone pour l'évolution sur le long terme de la zone tampon ont donc été annihilés. De la même manière, le passage de ces cyclones a finalement entraîné la construction d'un nombre important d'ouvrages de défense, qui sur le long terme vont continuer à dégrader les zones tampons. 2) La mise en place de pratiques pour restaurer les échanges sédimentaires au sein des cellules hydrosédimentaires. Des pratiques tel que le by-pass peuvent contribuer à conserver la plage des Roches Noires à Saint-Gilles, par exemple. 3) La multiplication des études pour mieux comprendre les processus en action et leurs échelles spatio-temporelles. L'étude de l'évolution de ces systèmes à différentes échelles temporelles (échelle pluri-décennale, impacts d'un événement, résilience) est fondamentale pour cartographier cette fonction de protection d'une part, et pour mieux comprendre les causes de sa dégradation d'autre part. Dans le cas de l'érosion par exemple, savoir si elle résulte d'une tendance naturelle de long terme ou si elle a été déclenchée par l'homme n'entraîne pas les mêmes réponses [Cooper et Mckenna, 2008]. 4) Enfin, la prise en compte dans la réflexion de l'ensemble des écosystèmes (récifs coralliens, herbiers, etc.), qui tous, en bon état de santé, contribueront à protéger les sociétés humaines sur le long terme. Dans certains cas, cette stratégie devra très certainement s'accompagner de la relocalisation des enjeux les plus exposés et les plus perturbateurs des processus naturels.

\section{CONCLUSIONS}

Le retour d'expérience de ces deux études souligne le rôle important de la dégradation des zones tampons dans l'augmentation de la vulnérabilité des enjeux humains face aux risques liés à la mer. Un enjeu majeur pour le futur est donc le maintien et/ou la restauration de ces systèmes, lorsque leur résilience est encore effective. Ces exemples traduisent également la faible intégration actuelle des processus naturels dans la gestion des risques, et ce dans les phases de prévention, de protection mais également de reconstruction. Cela se traduit par une échelle d'intervention inadaptée (souvent celle des individus), alors qu'une réflexion à l'échelle de la cellule sédimentaire est un prérequis. En parallèle, alors que les réponses naturelles aux perturbations sont variées, il apparaît nécessaire d'apporter des solutions différenciées et adaptées à chaque situation locale. L'étude des zones tampons est une nécessité dans la compréhension des conditions favorisant la résilience, afin d'engager une politique d'adaptation aux risques futurs, dans le contexte du changement climatique.

\section{REMERCIEMENTS}

Cette étude a bénéficié du soutien du Ministère de l'Environnement (MEEM) et de l'Agence Nationale de la Recherche (ANR) dans le cadre des projets de recherches Réomers (Programme Risques, Décision et Territoires) et Storisk (ANR-15-CE03-000302). Les images et photographies aériennes de Tubuai ont été fournies par le Service de l'Urbanisme de Polynésie française, que nous remercions chaleureusement. Nous remercions également Fanny Rubia, Adrien Prenveille, Paul-Bernard Rivet et Toanui Variamu pour la collecte de données sur le terrain et l'analyse de ces données, dans le cadre de leurs stages de Master.

\section{REFERENCES}

Arkema K., Guannel G., Verutes G., Wood S., Guerry A., Ruckelshaus M., Kareiva P., Lacayo M. (2013). - Coastal habitats shield people and property from sea-level rise and storms. Nature Climate Change, 3(10):913-918.

Aunay B., Bès de Berc S., Chateauminois E., Rey A., Thirard G., Vincent C. (2014). - Impacts du cyclone Bejisa sur l'île de la Réunion. Compte rendu des observations réalisées en janvier 2014. Rapport BRGM/RP-63149-FR. 93p. 
Berkes F. (2007). - Understanding uncertainty and reducing vulnerability: lessons from resilience thinking. Nat. Hazards, 41:283-295.

Cooper J. et Mckenna J. (2008). - Working with natural processes: The challenge for coastal protection strategies. Geographical Journal, 174(4):315-331.

Cazes-Duvat V. et Paskoff R. (2004). - Les littoraux des Mascareignes entre nature et aménagement. L'Harmattan, Paris, $187 \mathrm{p}$.

De Scally F. (2014). - Evaluation of storm surge risk: A case study from Rarotonga, Cook Islands. International Journal of Disaster Risk Reduction, 7:9-27.

Debaine F., et Robin M. (2012). - A new GIS modelling of coastal dune protection services against physical coastal hazards. Ocean and Coastal Management. 63:43-54.

Duvat V.K.E. et Magnan A.K. (2016). - Lessons from coastal risks governance on Reunion Island, Indian Ocean, France. In Facing Hydro-meteorological extremes events in Europe: a governance issue. Wiley-Blackwell.

Duvat V.K.E., Magnan A.K., Etienne S., Salmon C., Pignon-Mussaud C. (2016a). - Assessing the impacts of and resilience to Tropical Cyclone Bejisa, Reunion Island (Indian Ocean). Natural Hazards, 83(1):601-640.

Duvat V., Salmon C., Magnan A. (2016b). - Trajectoires de vulnérabilité des littoraux de l'île de la Réunion aux risques liés à la mer (1950-actuel). STUDY IDDRI, 4(16):172p.

Ellif C. et Silva I. (in press). - Coral reefs as the first line of defence: Shoreline protection in face of climate change. Marine Environmental Research, 1-7.

Ellison J., Mosley A., Helman M. (2017). - Assessing atoll shoreline condition to guide community management. Ecological indicators, 75:321-330.

Etienne S. (2012). - Marine inundation hazards in French Polynesia: geomorphic impacts of Tropical Cyclone Oli in February 2010. Geological Society, London, Special Publications, 361(1):21-39.

Laurent V. et Varney P. (2014). - Historique des cyclones de Polynésie française de 1831 à 2010. Météo-France. 172p.

Larrue S. et Chiron T. (2010). - Les îles de Polynésie française face à l'aléa cyclonique. Vertigo, 10(3)

Lecacheux S., Bulteau T., Pedreros R., Delvallée E., Paris F. (2013). - Projet ARAI 3 : Evaluation probabiliste des houles et des surcotes cycloniques en Polynésie française. Rapport BRGM/RP-61888-FR, 122p.

Rao N., Ghermandi A., Portela R., Wang X. (2015). - Global values of coastal ecosystem services: A spatial economic analysis of shoreline protection values. Ecosystem services, 11:95-105.

Spalding M., Ruffo S., Lacambra C., Meliane I., Hale LZ., Shepard C., Beck M. (2014). The role of ecosystems in coastal protection: Adapting to climate change and coastal hazards. Ocean \& Coastal Management, 90:50-57.

Troadec R. (2003). - Analyse de la prise en consideration du phénomène d'érosion affectant les plages coralliennes à la Réunion et proposition d'actualisation des techniques d'entretien de ces plages. Rapport du LSTUR, 20p.

Walker B., Holling C.S., Carpenter S.R., Kinzig A. (2004). - Resilience, Adaptability and Transformability in Socialecological Systems. Ecology and Society, 9(2):9p. 